\title{
THEORETICAL JUSTIFICATION OF VENTCEL'S BOUNDARY CONDITIONS FOR A THIN LAYER THREE-DIMENSIONAL THERMOELASTICITY PROBLEM
}

\author{
NASSIM BOUDRAHEM AND AHMED BERBOUCHA
}

Received 26 January, 2021

\begin{abstract}
In this paper, we consider a model of linear thermoelasticity for an elastic threedimensional body with high thermal conductivity covered by a thin layer on one of its faces. We show that Ventcel's boundary conditions may be obtained, as the thickness of the rigid body goes to zero.
\end{abstract}

2010 Mathematics Subject Classification: 35M10; 35G15; 35A15

Keywords: Ventcel's boundary conditions, Dirichlet conditions, mixed problem, thin layer, asymptotic analysis

\section{INTRODUCTION AND STATEMENT OF THE PROBLEM}

It is well known that there are two main approaches for the study of problems involving thin layers. We can consider directly the thin layers problems and use adapted numerical methods (see, for example [2,10,15]), or we incorporate the thin layer effect through an approximate boundary condition in an approximate way, see for example $[5,8,13]$ and the references therein. The second approach will be illustrated in this work by the study of a three-dimensional thermoelasticity coupled system for an elastic body covered on one of its faces by a thin layer or a thin shell of thickness $\varepsilon$.

More precisely, let us consider a three-dimensional elastic body with high thermal conductivity and occupying the set $\overline{\Omega_{+}}=[0,1] \times[0,1] \times[0,1]$. The boundary of $\Omega_{+}$is denoted by $\partial \Omega_{+}=\bar{\Sigma} \cup \Gamma_{+}$, where $\left.\Sigma=\right] 0,1[\times] 0,1[\times\{0\}$. The body is clamped on the portion $\Gamma_{+}$of its boundary and reinforced by a thin shell $\overline{\Omega_{-}^{\varepsilon}}=[0,1] \times[0,1] \times[-\varepsilon, 0]$, with $\varepsilon>0$ sufficiently small, on the other part $\Sigma$. The boundary of $\Omega_{-}^{\varepsilon}$ is denoted $\partial \Omega_{-}^{\varepsilon}=\overline{\Sigma^{\varepsilon}} \cup \bar{\Sigma} \cup \overline{\Gamma_{-}^{\varepsilon}}$, where $\left.\Sigma^{\varepsilon}=\right] 0,1[\times] 0,1\left[\times\{-\varepsilon\}\right.$ and the whole domain is $\Omega^{\varepsilon}=$ $\Omega_{+} \cup \Omega_{-}^{\varepsilon} \cup \Sigma$.

In what follows, the variables $u=\left(u_{1}, u_{2}, u_{3}\right)$ and $\theta$ represent respectively, a displacement vector field and the temperature. The functions $f=\left(f_{1}, f_{2}, f_{3}\right)$ and $g$ are the volume forces exerted on the body $\Omega^{\varepsilon}$. We also denote by $w^{\prime}, w^{\prime \prime}$ for the time 
derivatives of a function $w$. We then consider the following transmission system:

$$
\begin{cases}u^{\prime \prime}-\frac{1}{p} \operatorname{div}(p \nabla u)+\alpha \nabla \theta=f & \text { in } \Omega^{\varepsilon} \times(0, T) \\ \theta^{\prime}-\frac{1}{p} \operatorname{div}(p \nabla \theta)+\alpha \operatorname{div}\left(u^{\prime}\right)=g & \text { in } \Omega^{\varepsilon} \times(0, T)\end{cases}
$$

with Dirichlet conditions on the portion of the boundary $\Gamma_{+} \cup \Gamma_{-}^{\varepsilon}$

$$
u=0, \quad \theta=0 \quad \text { on }\left(\Gamma_{+} \cup \Gamma_{-}^{\varepsilon}\right) \times(0, T) .
$$

The boundary conditions on the face $\Sigma^{\varepsilon}$ are given by

$$
-p \partial_{v} u=0, \quad-p \partial_{v} \theta+\alpha p \gamma_{n}\left(u^{\prime}\right)=0 \quad \text { on } \Sigma^{\varepsilon} \times(0, T)
$$

where $\partial_{v}$ (respectively, $\gamma_{n}$ ) stands for the normal derivative (respectively, normal trace) operator. Here, $\alpha>0$ is a coupling parameter and $p$ is a discontinuous function on $\Sigma$, defined by

$$
p= \begin{cases}1 & \text { on } \Omega_{+}, \\ 1 / \varepsilon & \text { on } \Omega_{-}^{\varepsilon} .\end{cases}
$$

We define also the transmission conditions through $\Sigma$ by

$$
[u]=0, \quad[\theta]=0, \quad\left[-p \partial_{v} u\right]=0, \quad\left[-p \partial_{v} \theta+\alpha p \gamma_{n}\left(u^{\prime}\right)\right]=0, \text { on } \Sigma \times(0, T)
$$

where [.] denotes the skip function through the transmission surface $\Sigma$. We associate with the system (1.1) the initial conditions

$$
u(0)=u^{0}, \quad u^{\prime}(0)=u^{1}, \quad \theta(0)=\theta^{0} \quad \text { in } \Omega^{\varepsilon}
$$

where $u^{0}, u^{1}$ and $\theta^{0}$ are respectively the initial states of the displacement vector, the movement speed and the function of heat dissipation.

Recently, numerical modeling of thermoelasticity problems in a three dimensional region have been studied in [27] and [28]. However, in our case and due to the small thickness of the layer, these numerical computations can become cumbersome, especially for three-dimensional problems. So, our aim is to derive and justify approximate boundary conditions which replace the effect of the thin layer at the junction interface.

The analysis of such kind of problems in the two-dimensional case can be found in $[4,6,11,12,16,22-25]$. Let us mention the works $[1,3,7,14]$ in the three-dimensional case.

The paper is organized as follows. In Section 2, we show that Problem (1.1)-(1.5) admits a weak solution $(u, \theta)$ in a sense that we will specify later on. In Section 3, we construct an approximate problem depending on the thickness $\varepsilon$ of the thin layer by scaling argument. In Section 4, we establish a priori estimates which allow us to extract a weakly convergent subsequence. Finally, in Section 5 we show that the weak limit of the obtained approximate problem is a solution to a problem with Ventcel's boundary conditions. 


\section{Resolution of Problem (1.1)-(1.5)}

In this section, we will show that under some assumptions, Problem (1.1)-(1.5) admits a solution in a weak sense that will be specified later on. So, we will need some Sobolev spaces, which we recall in the following definitions:

$$
\mathcal{W}\left(\Omega^{\varepsilon}\right)=\left\{v \in \mathcal{H}^{1}\left(\Omega^{\varepsilon}\right) \mid v=0 \text { on } \Gamma_{+} \cup \Gamma_{-}^{\varepsilon}\right\}
$$

with

$$
\mathcal{H}^{1}\left(\Omega^{\varepsilon}\right)=\left\{v \in \mathcal{L}^{2}\left(\Omega^{\varepsilon}\right) \mid \partial_{x} v \in \mathcal{L}^{2}\left(\Omega^{\varepsilon}\right), \partial_{y} v \in \mathcal{L}^{2}\left(\Omega^{\varepsilon}\right), \partial_{z} v \in \mathcal{L}^{2}\left(\Omega^{\varepsilon}\right)\right\}
$$

where

$$
\mathcal{L}^{2}\left(\Omega^{\varepsilon}\right)=\left\{v=\left(v_{1}, v_{2}, v_{3}\right) / v_{i} \in L^{2}\left(\Omega^{\varepsilon}\right) \mid i=1,2,3\right\}
$$

and

$$
W\left(\Omega^{\varepsilon}\right)=\left\{\varphi \in H^{1}\left(\Omega^{\varepsilon}\right) \mid \varphi=0 \text { on } \Gamma_{+} \cup \Gamma_{-}^{\varepsilon}\right\}
$$

with

$$
H^{1}\left(\Omega^{\varepsilon}\right)=\left\{\varphi \in L^{2}\left(\Omega^{\varepsilon}\right) \mid \varphi^{\prime} \in L^{2}\left(\Omega^{\varepsilon}\right)\right\} .
$$

Lemma 1. $\mathcal{W}\left(\Omega^{\varepsilon}\right)$ and $W\left(\Omega^{\varepsilon}\right)$ are Hilbert spaces.

Proof. $\Gamma_{+} \cup \Gamma_{-}^{\varepsilon}$ is a piece of the boundary $\partial \Omega^{\varepsilon}$ of nonzero measure, the two spaces $\mathcal{W}\left(\Omega^{\varepsilon}\right)$ and $W\left(\Omega^{\varepsilon}\right)$ respectively with norms $\|\nabla v\|_{\mathcal{L}^{2}\left(\Omega^{\varepsilon}\right)}$ and $\|\nabla \varphi\|_{L^{2}\left(\Omega^{\varepsilon}\right)}$ are Hilbert spaces, see ([20], Chapter 1, paragraph 1.8).

\subsection{Weak formulation}

By a formal calculation and assuming that the Green formula

$$
\langle\operatorname{div}(u), \varphi\rangle_{\Omega^{\varepsilon}}=-\langle u, \nabla \varphi\rangle_{\Omega^{\varepsilon}}+\left\langle\gamma_{n}(u), \gamma_{0}(\varphi)\right\rangle_{\partial \Omega^{\varepsilon}}
$$

holds, we obtain a weak formulation $\left(P^{*}\right)$ of the problem in the form

$$
\begin{aligned}
& \frac{d}{d t}\left(\left\langle p \partial_{t} u, v\right\rangle_{\Omega^{\varepsilon}}\right)+\langle p \nabla u, \nabla v\rangle_{\Omega^{\varepsilon}}+\frac{d}{d t}\langle p \theta, \varphi\rangle_{\Omega^{\varepsilon}} \\
+ & \langle p \nabla \theta, \nabla \varphi\rangle_{\Omega^{\varepsilon}}+\alpha\langle p \nabla \theta, v\rangle_{\Omega^{\varepsilon}}-\alpha\left\langle p \partial_{t} u, \nabla \varphi\right\rangle_{\Omega^{\varepsilon}}=\langle p f, v\rangle_{\Omega^{\varepsilon}}+\langle p g, \varphi\rangle_{\Omega^{\varepsilon}}
\end{aligned}
$$

with

$$
\begin{aligned}
\langle p u(0, .), v\rangle_{\Omega^{\varepsilon}} & =\left\langle p u^{0}, v\right\rangle_{\Omega^{\varepsilon}} \\
\frac{d}{d t}\langle p u, v\rangle_{\Omega^{\varepsilon}}(0, .) & =\left\langle p u^{1}, v\right\rangle_{\Omega^{\varepsilon}} \\
\langle p \theta, \varphi\rangle_{\Omega^{\varepsilon}}(0, .) & =\left\langle p \theta^{0}, \varphi\right\rangle_{\Omega^{\varepsilon}}
\end{aligned}
$$

for all $(v, \varphi) \in \mathcal{W}\left(\Omega^{\varepsilon}\right) \times W\left(\Omega^{\varepsilon}\right)$. 


\subsection{Existence and uniqueness of a weak solution}

Proposition 1. Let us assume that

$$
\begin{gathered}
f \in L^{2}\left(0, T ; \mathcal{L}^{2}\left(\Omega^{\varepsilon}\right)\right), \quad g \in L^{2}\left(0, T ; L^{2}\left(\Omega^{\varepsilon}\right),\right. \\
u^{0} \in \mathcal{W}\left(\Omega^{\varepsilon}\right), \quad u^{1} \in \mathcal{L}^{2}\left(\Omega^{\varepsilon}\right), \quad \theta^{0} \in W\left(\Omega^{\varepsilon}\right),
\end{gathered}
$$

then the weak problem (2.1)-(2.4) admits a unique solution $(u, \theta)$ satisfying

$$
\begin{gathered}
u \in L^{\infty}\left(0, T ; \mathcal{W}\left(\Omega^{\varepsilon}\right)\right), \quad \partial_{t} u \in L^{\infty}\left(0, T ; \mathcal{L}^{2}\left(\Omega^{\varepsilon}\right)\right), \\
\theta \in L^{\infty}\left(0, T ; L^{2}\left(\Omega^{\varepsilon}\right)\right) \cap L^{2}\left(0, T ; W\left(\Omega^{\varepsilon}\right)\right) .
\end{gathered}
$$

Proof. We will demonstrate the above proposition in three steps. First, we establish a priori estimates which will allow us at second time through the Faedo-Galerkin method to construct convergent sequences to a solution of our problem in the weak sense and finally we establish uniqueness.

Step 1: A priori estimate

Let us now consider the energy function, see the definition e.g. in $[17,19,21]$ and references therein. Then, we denote by $E(t)$ the energy of the system for $t \neq 0$

$$
E(t)=\frac{1}{2}\left(\left\|\partial_{t} u\right\|_{\Omega_{+}}^{2}+\frac{1}{\varepsilon}\left\|\partial_{t} u\right\|_{\Omega_{-}^{\varepsilon}}^{2}+\|\nabla u\|_{\Omega_{+}}^{2}+\frac{1}{\varepsilon}\|\nabla u\|_{\Omega_{-}^{\varepsilon}}^{2}+\|\theta\|_{\Omega_{+}}^{2}+\frac{1}{\varepsilon}\|\theta\|_{\Omega_{-}^{\varepsilon}}^{2}\right)
$$

then we set

$$
E(0)=\frac{1}{2}\left(\left\|u^{1}\right\|_{\Omega_{+}}^{2}+\frac{1}{\varepsilon}\left\|u^{1}\right\|_{\Omega_{-}^{\varepsilon}}^{2}+\left\|\nabla u^{0}\right\|_{\Omega_{+}}^{2}+\frac{1}{\varepsilon}\left\|\nabla u^{0}\right\|_{\Omega_{-}^{\varepsilon}}^{2}+\left\|\theta^{0}\right\|_{\Omega_{+}}^{2}+\frac{1}{\varepsilon}\left\|\theta^{0}\right\|_{\Omega_{-}^{\varepsilon}}^{2}\right) .
$$

It suffices then to take in the weak problem (2.1) - (2.4) $v=\partial_{t} u$ and $\varphi=\theta$ to obtain the so-called energy equality

$$
\begin{gathered}
E(t)+\int_{0}^{t}\left(\|\nabla \theta\|_{\Omega_{+}}^{2}+\frac{1}{\varepsilon}\|\nabla \theta\|_{\Omega_{-}^{\varepsilon}}^{2}\right) d s= \\
E(0)+\int_{0}^{t}\left(\left\langle f, \partial_{t} u\right\rangle_{\Omega_{+}}+\left\langle f, \partial_{t} u\right\rangle_{\Omega_{-}^{\varepsilon}}+\langle g, \theta\rangle_{\Omega_{+}}+\langle g, \theta\rangle_{\Omega_{-}^{\varepsilon}}\right) d s .
\end{gathered}
$$

In virtue of Cauchy-Schwarz inequality, we establish an estimate of the energy in function of the initial conditions and data of the problem

$$
E(t) \leq c\left(E(0)+\int_{0}^{t}\left(\|f\|_{\Omega^{\varepsilon}}^{2}+\|g\|_{\Omega^{\varepsilon}}^{2}\right) d s\right) \quad \forall t \in(0, T)
$$

where $c$ is a positive constant. Therefore, thanks to the assumptions of the proposition, the energy of the system is bounded in time

$$
E(t) \leq c \quad \forall t \in(0, T)
$$


where $c$ is another positive constant which depends only on the data. This also gives an a priori estimate of $\theta$ in $L^{2}\left(0, T ; W\left(\Omega^{\varepsilon}\right)\right)$. Indeed, we have

$$
\int_{0}^{T}\left(\|\nabla \theta\|_{\Omega_{+}}^{2}+\frac{1}{\varepsilon}\|\nabla \theta\|_{\Omega_{-}^{\varepsilon}}^{2}\right) d s \leq c
$$

where $c$ is another positive constant which depends only on the data.

Step 2: Existence - Faedo-Galerkin method.

$\mathcal{W}\left(\Omega^{\varepsilon}\right)$ and $W\left(\Omega^{\varepsilon}\right)$ are separable Hilbert spaces. Let $\left(v_{i}\right)_{i}$ (respectively, $\left.\left(\varphi_{i}\right)_{i}\right)$ a basis of $\mathcal{W}\left(\Omega^{\varepsilon}\right)$ (respectively, $W\left(\Omega^{\varepsilon}\right)$ ). We introduce then the approximate solution $\left(u_{m}(t), \theta_{m}(t)\right)$ defined by

$$
u_{m}(t)=\sum_{i=1}^{m} h_{i m}(t) v_{i} \quad \text { and } \quad \theta_{m}(t)=\sum_{i=1}^{m} k_{i m}(t) \varphi_{i}
$$

which has to satisfy the approximate problem

$$
\begin{aligned}
& \frac{d}{d t}\left(\left\langle p \partial_{t} u_{m}, v_{j}\right\rangle_{\Omega^{\varepsilon}}\right)+\left\langle p \nabla u_{m}, \nabla v_{j}\right\rangle_{\Omega^{\varepsilon}}+\frac{d}{d t}\left\langle p \theta_{m}, \varphi_{j}\right\rangle_{\Omega^{\varepsilon}}+\left\langle p \nabla \theta_{m}, \nabla \varphi_{j}\right\rangle_{\Omega^{\varepsilon}} \\
& +\alpha\left\langle p \nabla \theta_{m}, v_{j}\right\rangle_{\Omega}-\alpha\left\langle p \partial_{t} u_{m}, \nabla \varphi_{j}\right\rangle_{\Omega^{\varepsilon}}=\left\langle p f, v_{j}\right\rangle_{\Omega^{\varepsilon}}+\left\langle p g, \varphi_{j}\right\rangle_{\Omega^{\varepsilon}}
\end{aligned}
$$

with

$$
\left\{\begin{array}{l}
u_{m}(0)=u^{0 m}=\sum_{j=1}^{m} h_{j m}(0) v_{j}, \quad \partial_{t} u_{m}(0)=u^{1 m}=\sum_{j=1}^{m} h_{j m}^{\prime}(0) v_{j}, \\
\theta_{m}(0)=\theta^{0 m}=\sum_{j=1}^{m} k_{j m}(0) \varphi_{j},
\end{array}\right.
$$

for all $j=1, \ldots, m$. The coefficients $h_{j m}(0)$ and $k_{j m}(0)$ will be chosen such that

$$
u^{0 m} \rightarrow u^{0} \text { in } \mathcal{W}\left(\Omega^{\varepsilon}\right), \quad u^{1 m} \rightarrow u^{1} \text { in } \mathcal{L}^{2}\left(\Omega^{\varepsilon}\right) \quad \text { and } \theta^{0 m} \rightarrow \theta^{0} \text { in } W\left(\Omega^{\varepsilon}\right) .
$$

For each $j=1, \ldots, m$, the system (2.8) is a system of ordinary differential equations with initial Cauchy conditions (2.9). So, the system (2.8)-(2.9) of the unknowns $h_{j m}(t)$ and $k_{j m}(t)$ has a unique solution, see for example [26]. Note that Estimates (2.6) and (2.7) remain valid for the approximate solutions, then

$$
\left\|\partial_{t} u_{m}\right\|_{\Omega_{+}}^{2}+\frac{1}{\varepsilon}\left\|\partial_{t} u_{m}\right\|_{\Omega_{-}^{\varepsilon}}^{2} \leq c, \quad \quad\left\|\nabla u_{m}\right\|_{\Omega_{+}}^{2}+\frac{1}{\varepsilon}\left\|\nabla u_{m}\right\|_{\Omega_{-}^{\varepsilon}}^{2} \leq c
$$

and

$$
\left\|\theta_{m}\right\|_{\Omega_{+}}^{2}+\frac{1}{\varepsilon}\left\|\theta_{m}\right\|_{\Omega_{-}^{\varepsilon}}^{2} \leq c, \quad \int_{0}^{T}\left(\left\|\nabla \theta_{m}\right\|_{\Omega_{+}}^{2}+\frac{1}{\varepsilon}\left\|\nabla \theta_{m}\right\|_{\Omega_{-}^{\varepsilon}}^{2}\right) d s \leq c
$$

where $c$ denotes various constants independent of $m$. This means that

$$
\left(u_{m}, \theta_{m}\right) \text { is bounded in } L^{2}\left(0, T ; \mathcal{W}\left(\Omega^{\varepsilon}\right)\right) \times L^{2}\left(0, T ; W\left(\Omega^{\varepsilon}\right)\right) .
$$

So, we can extract a subsequence from $\left(u_{m}, \theta_{m}\right)$ (that we continue to denote $\left(u_{m}, \theta_{m}\right)$ ) such that,

$$
u_{m} \rightarrow u \text { weakly in } L^{2}\left(0, T ; \mathcal{W}\left(\Omega^{\varepsilon}\right)\right)
$$


and

$$
\theta_{m} \rightarrow \theta \text { weakly in } L^{2}\left(0, T ; W\left(\Omega^{\varepsilon}\right)\right) .
$$

As inequality (2.5) is still valid for the new subsequence $u_{m}$, then $\partial_{t} u_{m}$ is bounded in $L^{2}\left(0, T ; \mathcal{L}^{2}\left(\Omega^{\varepsilon}\right)\right)$. So, it is possible to extract a subsequence denoted again $u_{m}$ such that $\partial_{t} u_{m}$ converges weakly to some $\chi$ in $L^{2}\left(0, T ; \mathcal{L}^{2}\left(\Omega^{\varepsilon}\right)\right)$. We deduce that $\chi=\partial_{t} u$ almost everywhere because the equality holds in the distributional sense. Then,

$$
\partial_{t} u_{m} \rightarrow \partial_{t} u \text { weakly in } L^{2}\left(0, T ; \mathcal{L}^{2}\left(\Omega^{\varepsilon}\right)\right) .
$$

It remains to show that $(u, \theta)$ thus constructed is the unique solution of Problem (2.1)-(2.4). For this purpose, we multiply the equation (2.10) by a test function $\psi \in \mathcal{D}(0, T)$, the space of infinitely differentiable functions with compact support in $(0, T)$, and then we integrate with respect to the time variable and we obtain

$$
\begin{array}{r}
\int_{0}^{T} \frac{d}{d t}\left(\left\langle p \partial_{t} u_{m}, v_{j}\right\rangle_{\Omega^{\varepsilon}}\right) \psi d s+\int_{0}^{T}\left\langle p \nabla u_{m}, \nabla v_{j}\right\rangle_{\Omega^{\varepsilon}} \psi d s+\int_{0}^{T} \frac{d}{d t}\left\langle p \theta_{m}, \varphi_{j}\right\rangle_{\Omega^{\varepsilon}} \psi d s \\
+\int_{0}^{T}\left\langle p \nabla \theta_{m}, \nabla \varphi_{j}\right\rangle_{\Omega^{\varepsilon}} \psi d s+\alpha \int_{0}^{T}\left\langle p \nabla \theta_{m}, v_{j}\right\rangle_{\Omega^{\varepsilon}} \psi d s-\alpha \int_{0}^{T}\left\langle p \partial_{t} u_{m}, \nabla \varphi_{j}\right\rangle_{\Omega^{\varepsilon}} \psi d s \\
=\int_{0}^{T}\left\langle p f, v_{j}\right\rangle_{\Omega^{\varepsilon}} \psi d s+\int_{0}^{T}\left\langle p g, \varphi_{j}\right\rangle_{\Omega^{\varepsilon}} \psi d s
\end{array}
$$

for all $j=1, \ldots, m$. Integrating by parts with respect to the time variable, we obtain

$$
\begin{gathered}
\int_{0}^{T}\left\langle p u_{m}, v_{j}\right\rangle_{\Omega^{\varepsilon}} \psi^{\prime \prime} d s+\int_{0}^{T}\left\langle p \nabla u_{m}, \nabla v_{j}\right\rangle_{\Omega^{\varepsilon}} \psi d s-\int_{0}^{T}\left\langle p \theta_{m}, \varphi_{j}\right\rangle_{\Omega^{\varepsilon}} \psi^{\prime} d s \\
+\int_{0}^{T}\left\langle p \nabla \theta_{m}, \nabla \varphi_{j}\right\rangle_{\Omega^{\varepsilon}} \psi d s+\alpha \int_{0}^{T}\left\langle p \nabla \theta_{m}, v_{j}\right\rangle_{\Omega^{\varepsilon}} \psi d s+\alpha \int_{0}^{T}\left\langle p u_{m}, \nabla \varphi_{j}\right\rangle_{\Omega^{\varepsilon}} \psi^{\prime} d s \\
=\int_{0}^{T}\left\langle p f, v_{j}\right\rangle_{\Omega^{\varepsilon}} \psi d s+\int_{0}^{T}\left\langle p g, \varphi_{j}\right\rangle_{\Omega^{\varepsilon}} \psi d s
\end{gathered}
$$

for all $j=1, \ldots, m$. Summing over $j$, and making the passage to the limit, the previous equality remains true for all $v \in \mathcal{W}\left(\Omega^{\varepsilon}\right)$ and for all $\varphi \in W\left(\Omega^{\varepsilon}\right)$. By density arguments, the previous equality remains true for all $v \in L^{2}\left(0, T ; \mathcal{W}\left(\Omega^{\varepsilon}\right)\right)$ and for all $\varphi \in L^{2}\left(0, T ; W\left(\Omega^{\varepsilon}\right)\right)$, see for example[18]. Passing to the limit and using (2.10), (2.11) and (2.12), $(u, \theta)$ is a weak solution of the Problem (2.1)-(2.4).

Step 3: Uniqueness

For uniqueness, we set

$$
f=g=u^{0}=u^{1}=0, \quad \text { and } \theta^{0}=0
$$

in Problem (2.1)-(2.4). Thanks to inequality (2.5), we obtain

$$
E(t)=0 \quad \forall t \in(0, T) .
$$

This implies that

$$
\theta=0 \text { on } \Omega_{+} \quad \text { and } \theta=0 \text { on } \Omega_{-}^{\varepsilon}
$$


and in virtue of the transmission condition (1.4), it follows that

$$
\theta=0 \text { on } \Omega^{\varepsilon} .
$$

Similarly, we can show that

$$
\nabla u=0 \text { on } \Omega_{+} \quad \text { and } \nabla u=0 \text { on } \Omega_{-}^{\varepsilon} .
$$

As $\|\nabla u\|$ is an equivalent norm to the norm of $\mathcal{W}\left(\Omega^{\varepsilon}\right)$, it follows that

$$
u=0 \text { on } \Omega_{+} \quad \text { and } u=0 \text { on } \Omega_{-}^{\varepsilon} .
$$

Finally, thanks to the transmission condition (1.4), we have

$$
u=0 \text { on } \Omega^{\varepsilon} .
$$

\section{SCALED PROBLEM}

\subsection{Scaling}

In order to study the asymptotic behavior of the solution of Problem (2.1)-(2.4) as $\varepsilon \rightarrow 0$, and since $\Omega^{\varepsilon}$ vary with $\varepsilon$, we first transform the body $\Omega^{\varepsilon}$ into a fixed body (independent of $\varepsilon$ ) by means of a change of scaling. Then, the displacement field $u$ and the heat propagation $\theta$ can be expressed in function of $\varepsilon$. So, let us consider the following change of variable $T_{\varepsilon}: \mathbb{R}^{3} \rightarrow \mathbb{R}^{3}$ defined by

$$
T_{\varepsilon}(x, y, z)= \begin{cases}(x, y, z) & \text { if } z \geq 0 \\ (x, y, \varepsilon z) & \text { if } z<0\end{cases}
$$

which is a similarity operating on the $z$ variable and it makes stiff (independent of $\varepsilon$ ) the open domain $\Omega^{\varepsilon}$. We note then

$$
\begin{aligned}
\Omega & \left.=T_{\varepsilon}^{-1}\left(\Omega^{\varepsilon}\right)=\right] 0.1\left[^{2} \times\right]-1,1[, & & \\
\Omega_{+} & =T_{\varepsilon}^{-1}\left(\Omega_{+}\right), & & \left.\Omega_{-}=T_{\varepsilon}^{-1}\left(\Omega_{-}^{\varepsilon}\right)=\right] 0.1\left[^{2} \times\right]-1,0[, \\
\Sigma & =T_{\varepsilon}^{-1}(\Sigma), & & \left.\Sigma_{-}=T_{\varepsilon}^{-1}\left(\Sigma^{\varepsilon}\right)=\right] 0.1\left[^{2} \times\{-1\},\right. \\
\Gamma_{+} & =T_{\varepsilon}^{-1}\left(\Gamma_{+}\right), & & \Gamma_{-}=T_{\varepsilon}^{-1}\left(\Gamma_{-}^{\varepsilon}\right) .
\end{aligned}
$$

The transformation $T_{\varepsilon}$ permits also to define the scaling of the state variables as follows: to the scalar function $\varphi$, we associate the function

$$
\varphi^{\varepsilon}=\varphi \circ T_{\varepsilon}
$$

and to the vector field $v$, we associate the field

$$
v^{\varepsilon}=T_{\varepsilon} \circ v \circ T_{\varepsilon} .
$$


This allows us to express the Sobolev spaces on the open set $\Omega$ by defining $\mathcal{W}^{\varepsilon}(\Omega)$ and $W^{\varepsilon}(\Omega)$ as follows:

$$
\begin{aligned}
& \mathcal{W}^{\varepsilon}(\Omega)=\left\{v_{ \pm}^{\varepsilon} \in \mathcal{H}^{1}\left(\Omega_{ \pm}\right) \mid[v]=0 \text { on } \Sigma \text { and } v=0 \text { on } \Gamma_{+} \cup \Gamma_{-}\right\} \\
& W^{\varepsilon}(\Omega)=\left\{\varphi \in H^{1}(\Omega) \mid[\varphi]=0 \text { on } \Sigma \text { and } \varphi=0 \text { on } \Gamma_{+} \cup \Gamma_{-}\right\} .
\end{aligned}
$$

We naturally endow $\mathcal{W}^{\varepsilon}(\Omega)$ and $W^{\varepsilon}(\Omega)$ by the standard norms $\|\nabla v\|_{\mathcal{L}^{2}\left(\Omega^{\varepsilon}\right)}$ and $\|\nabla \varphi\|_{L^{2}\left(\Omega^{\varepsilon}\right)}$, respectively. It is obvious that $\mathcal{W}^{\varepsilon}(\Omega)$ and $W^{\varepsilon}(\Omega)$ are Hilbert spaces for the simple reason that $v^{\varepsilon} \in \mathcal{W}^{\varepsilon}(\Omega)$ is equivalent to $v \in \mathcal{W}\left(\Omega^{\varepsilon}\right)$ and $\varphi^{\varepsilon} \in W^{\varepsilon}(\Omega)$ is equivalent to $\varphi \in W\left(\Omega^{\varepsilon}\right)$.

\subsection{Weak formulation in the fixed open set $\Omega$}

We will now set the variational problem (2.1)-(2.4) in the open set $\Omega$ and we associate to the data $f, g, u^{0}, u^{1}$ and $\theta^{0}$ of Problem (2.1)-(2.4) new data defined on the set $\Omega$ and obtained by changing of variables and scale, which we denote $f^{\varepsilon}, g^{\varepsilon}, u^{\varepsilon 0}, u^{\varepsilon 1}$ and $\theta^{\varepsilon 0}$, respectively.

Proposition 2. Under the assumptions of Proposition $1,\left(u^{\varepsilon}, \theta^{\varepsilon}\right)$ satisfies

$$
\begin{gathered}
u^{\varepsilon} \in L^{\infty}(0, T ; \mathcal{W}(\Omega)), \quad \partial_{t} u^{\varepsilon} \in L^{\infty}\left(0, T ; \mathcal{L}^{2}(\Omega)\right), \\
\text { and } \theta^{\varepsilon} \in L^{\infty}\left(0, T ; L^{2}(\Omega)\right) \cap L^{2}(0, T ; W(\Omega))
\end{gathered}
$$

and it is the unique solution of Problem $\left(P^{\varepsilon}\right)$ below

$$
\begin{aligned}
& \frac{d}{d t}\left(\left\langle\partial_{t} u^{\varepsilon}, v^{\varepsilon}\right\rangle_{+}+\left\langle\partial_{t} u_{1}^{\varepsilon}, v_{1}^{\varepsilon}\right\rangle_{-}+\left\langle\partial_{t} u_{2}^{\varepsilon}, v_{2}^{\varepsilon}\right\rangle_{-}+\frac{1}{\varepsilon^{2}}\left\langle\partial_{t} u_{3}^{\varepsilon}, v_{3}^{\varepsilon}\right\rangle_{-}\right) \\
& +\left\langle\nabla u^{\varepsilon}, \nabla v^{\varepsilon}\right\rangle_{+}+\left\langle\partial_{x} u_{1}^{\varepsilon}, \partial_{x} v_{1}^{\varepsilon}\right\rangle_{-}+\left\langle\partial_{y} u_{1}^{\varepsilon}, \partial_{y} v_{1}^{\varepsilon}\right\rangle_{-}+\frac{1}{\varepsilon^{2}}\left\langle\partial_{z} u_{1}^{\varepsilon}, \partial_{z} v_{1}^{\varepsilon}\right\rangle_{-} \\
& +\left\langle\partial_{x} u_{2}^{\varepsilon}, \partial_{x} v_{2}^{\varepsilon}\right\rangle_{-}+\left\langle\partial_{y} u_{2}^{\varepsilon}, \partial_{y} v_{2}^{\varepsilon}\right\rangle_{-} \frac{1}{\varepsilon^{2}}\left\langle\partial_{z} u_{2}^{\varepsilon}, \partial_{z} v_{2}^{\varepsilon}\right\rangle_{-}+\frac{1}{\varepsilon^{2}}\left\langle\partial_{x} u_{3}^{\varepsilon}, \partial_{x} v_{3}^{\varepsilon}\right\rangle_{-} \\
& +\frac{1}{\varepsilon^{2}}\left\langle\partial_{y} u_{3}^{\varepsilon}, \partial_{y} v_{3}^{\varepsilon}\right\rangle_{-}+\frac{1}{\varepsilon^{4}}\left\langle\partial_{z} u_{3}^{\varepsilon}, \partial_{z} v_{3}^{\varepsilon}\right\rangle_{-}+\frac{d}{d t}\left(\left\langle\theta^{\varepsilon}, \varphi^{\varepsilon}\right\rangle_{+}+\left\langle\theta^{\varepsilon}, \varphi^{\varepsilon}\right\rangle_{-}\right) \\
& +\left\langle\nabla \theta^{\varepsilon}, \nabla \varphi^{\varepsilon}\right\rangle_{+}+\left\langle\partial_{x} \theta^{\varepsilon}, \partial_{x} \varphi^{\varepsilon}\right\rangle_{-}+\left\langle\partial_{y} \theta^{\varepsilon}, \partial_{y} \varphi^{\varepsilon}\right\rangle_{-}+\frac{1}{\varepsilon^{2}}\left\langle\partial_{z} \theta^{\varepsilon}, \partial_{z} \varphi^{\varepsilon}\right\rangle_{-} \\
& +\alpha\left\langle\nabla \theta^{\varepsilon}, \vec{v}^{\varepsilon}\right\rangle_{+}+\alpha\left\langle\partial_{x} \theta^{\varepsilon}, v_{1}^{\varepsilon}\right\rangle_{-}+\alpha\left\langle\partial_{y} \theta^{\varepsilon}, v_{2}^{\varepsilon}\right\rangle_{-}+\frac{1}{\varepsilon^{2}} \alpha\left\langle\partial_{z} \theta^{\varepsilon}, v_{3}^{\varepsilon}\right\rangle_{-} \\
& -\alpha\left\langle\partial_{t} \vec{u}^{\varepsilon}, \nabla \varphi^{\varepsilon}\right\rangle_{+}-\alpha\left\langle\partial_{t} u_{1}^{\varepsilon}, \partial_{x} \varphi^{\varepsilon}\right\rangle_{-}-\alpha\left\langle\partial_{t} u_{2}^{\varepsilon}, \partial_{y} \varphi^{\varepsilon}\right\rangle_{-}-\frac{1}{\varepsilon^{2}} \alpha\left\langle\partial_{t} u_{3}^{\varepsilon}, \partial_{z} \varphi^{\varepsilon}\right\rangle_{-} \\
& =\left\langle f^{\varepsilon}, v^{\varepsilon}\right\rangle_{+}+\left\langle f_{1}^{\varepsilon}, v_{1}^{\varepsilon}\right\rangle_{-}+\left\langle f_{2}^{\varepsilon}, v_{2}^{\varepsilon}\right\rangle_{-}+\frac{1}{\varepsilon^{2}}\left\langle f_{3}^{\varepsilon}, v_{3}^{\varepsilon}\right\rangle_{-}+\left\langle g^{\varepsilon}, \varphi^{\varepsilon}\right\rangle_{+}+\left\langle g^{\varepsilon}, \varphi^{\varepsilon}\right\rangle_{-}
\end{aligned}
$$


with initial conditions

$$
\begin{aligned}
&\left\langle u^{\varepsilon}(0), v^{\varepsilon}\right\rangle_{+}+\left\langle u_{1}^{\varepsilon}(0), v_{1}^{\varepsilon}\right\rangle_{-}+\left\langle u_{2}^{\varepsilon}(0), v_{2}^{\varepsilon}\right\rangle_{-}+\frac{1}{\varepsilon^{2}}\left\langle u_{3}^{\varepsilon}(0), v_{3}^{\varepsilon}\right\rangle_{-} \\
&=\left\langle u^{\varepsilon 0}, v^{\varepsilon}\right\rangle_{+}+\left\langle u_{1}^{\varepsilon 0}, v_{1}^{\varepsilon}\right\rangle_{-}+\left\langle u_{2}^{\varepsilon 0}, v_{2}^{\varepsilon}\right\rangle_{-}+\frac{1}{\varepsilon^{2}}\left\langle u_{3}^{\varepsilon 0}, v_{3}^{\varepsilon}\right\rangle_{-} \\
&\left\langle\partial_{t} u^{\varepsilon}(0), v^{\varepsilon}\right\rangle_{+}+\left\langle\partial_{t} u_{1}^{\varepsilon}(0), v_{1}^{\varepsilon}\right\rangle_{-}+\left\langle\partial_{t} u_{2}^{\varepsilon}(0), v_{2}^{\varepsilon}\right\rangle_{-}+\frac{1}{\varepsilon^{2}}\left\langle\partial_{t} u_{3}^{\varepsilon}(0), v_{3}^{\varepsilon}\right\rangle_{-} \\
&=\left\langle u^{\varepsilon 1}, v^{\varepsilon}\right\rangle_{+}+\left\langle u_{1}^{\varepsilon 1}, v_{1}^{\varepsilon}\right\rangle_{-}+\left\langle u_{2}^{\varepsilon 1}, v_{2}^{\varepsilon}\right\rangle_{-}+\frac{1}{\varepsilon^{2}}\left\langle u_{3}^{\varepsilon 1}, v_{3}^{\varepsilon}\right\rangle_{-} \\
&\left\langle\theta^{\varepsilon}(0), \varphi^{\varepsilon}\right\rangle_{+}+\left\langle\theta^{\varepsilon}(0), \varphi^{\varepsilon}\right\rangle_{-}=\left\langle\theta^{\varepsilon 0}, \varphi^{\varepsilon}\right\rangle_{+}+\left\langle\theta^{\varepsilon 0}, \varphi^{\varepsilon}\right\rangle_{-}
\end{aligned}
$$

for all $\left(v^{\varepsilon}, \varphi^{\varepsilon}\right) \in \mathcal{W}^{\varepsilon}(\Omega) \times W^{\varepsilon}(\Omega)$, where $\langle., .\rangle_{ \pm}$denotes $\langle., .\rangle_{\Omega_{ \pm}}$.

Proof. This is a direct consequence of Proposition 1 by changing variable and scale.

\section{A PRIORI ESTIMATES AND THEIR CONSEQUENCES}

Proposition 3. Under the following assumptions:

(i) $E^{\varepsilon}(0)$ is bounded independently of $\varepsilon$,

(ii) $f_{+}^{\varepsilon}$ and $g_{+}^{\varepsilon}$ are bounded independently of $\varepsilon$ in $\mathcal{L}^{2}\left(\Omega_{+}\right)$and $L^{2}\left(\Omega_{+}\right)$, respectively,

(iii) $f_{1-}^{\varepsilon}, f_{2-}^{\varepsilon}, \frac{1}{\varepsilon} f_{3-}^{\varepsilon}$ and $g_{-}^{\varepsilon}$ are bounded independently of $\varepsilon$ in $L^{2}\left(\Omega_{-}\right)$,

we have the following a priori estimates:

$$
\begin{gathered}
\left\|\partial_{t} u^{\varepsilon}\right\|_{+}^{2} \leq c, \quad\left\|\partial_{t} u_{1}^{\varepsilon}\right\|_{-}^{2} \leq c, \quad\left\|\partial_{t} u_{2}^{\varepsilon}\right\|_{-}^{2} \leq c, \quad \frac{1}{\varepsilon^{2}}\left\|\partial_{t} u_{3}^{\varepsilon}\right\|_{-}^{2} \leq c, \\
\left\|\nabla u^{\varepsilon}\right\|_{+}^{2} \leq c, \quad\left\|\partial_{x} u_{1}^{\varepsilon}\right\|_{-}^{2} \leq c, \quad\left\|\partial_{y} u_{1}^{\varepsilon}\right\|_{-}^{2} \leq c, \quad \frac{1}{\varepsilon^{2}}\left\|\partial_{z} u_{1}^{\varepsilon}\right\|_{-}^{2} \leq c, \\
\left\|\partial_{x} u_{2}^{\varepsilon}\right\|_{-}^{2} \leq c, \quad\left\|\partial_{y} u_{2}^{\varepsilon}\right\|_{-}^{2} \leq c, \quad \frac{1}{\varepsilon^{2}}\left\|\partial_{z} u_{2}^{\varepsilon}\right\|_{-}^{2} \leq c \\
\frac{1}{\varepsilon^{2}}\left\|\partial_{x} u_{3}^{\varepsilon}\right\|_{-}^{2} \leq c, \quad \quad \frac{1}{\varepsilon^{2}}\left\|\partial_{y} u_{3}^{\varepsilon}\right\|_{-}^{2} \leq c, \quad \frac{1}{\varepsilon 4}\left\|\partial_{z} u_{3}^{\varepsilon}\right\|_{-}^{2} \leq c, \\
\int_{0}^{T}\left\|\nabla \theta^{\varepsilon}\right\|_{+}^{2} d s \leq c \quad\left\|\theta^{\varepsilon}\right\|_{+}^{2} \leq c, \quad \theta_{0}^{\varepsilon} \|_{-}^{2} \leq c \\
\int_{0}^{T}\left\|\partial_{y} \theta^{\varepsilon}\right\|_{-}^{2} d s \leq c, \quad \partial_{x} \theta^{\varepsilon} \|_{-}^{2} d s \leq c,
\end{gathered}
$$

where $c$ denotes various constants independent of $\varepsilon$. 
Proof. This is a direct consequence of the energy equality and the following inequality:

$$
E^{\varepsilon}(t) \leq c\left(E^{\varepsilon}(0)+\int_{0}^{T}\left(\left\|f^{\varepsilon}\right\|_{+}^{2}+\left\|f_{1}^{\varepsilon}\right\|_{-}^{2}+\left\|f_{2}^{\varepsilon}\right\|_{-}^{2}+\frac{1}{\varepsilon^{2}}\left\|f_{3}^{\varepsilon}\right\|_{-}^{2}+\left\|g^{\varepsilon}\right\|_{+}^{2}+\left\|g^{\varepsilon}\right\|_{-}^{2}\right) d s\right)
$$

established in the same way as in Proposition 1.

From the a priori estimates of Proposition 3, there exists a subsequence of $\left(u^{\varepsilon}, \theta^{\varepsilon}\right)$ which we denote again $\left(u^{\varepsilon}, \theta^{\varepsilon}\right)$, such that

$$
\begin{gathered}
u^{\varepsilon} \text { converges weakly-* in } L^{\infty}(0, T ; \mathcal{W}(\Omega)) \\
\partial_{t} u^{\varepsilon} \text { converges weakly-* in } L^{\infty}\left(0, T ; \mathcal{L}^{2}(\Omega)\right)
\end{gathered}
$$

$\theta^{\varepsilon}$ converges weakly-* in $L^{\infty}\left(0, T ; L^{2}(\Omega)\right)$ and converges weakly in $L^{2}(0, T ; W(\Omega))$. We define the following Hilbert spaces:

$$
\begin{aligned}
\mathcal{V}\left(\Omega_{+}\right) & =\left\{v \in \mathcal{H}^{1}\left(\Omega_{+}\right) \mid v=0 \text { on } \Gamma_{+}, v_{1} \in H_{0}^{1}(\Sigma), v_{2} \in H_{0}^{1}(\Sigma) \text { and } v_{3}=0 \text { on } \Sigma\right\} \\
\mathcal{H}^{1}\left(\Omega_{+}\right) & =\left\{v \in \mathcal{L}^{2}\left(\Omega_{+}\right)|v|_{\Sigma} \in \mathcal{L}^{2}(\Sigma)\right\} \\
V\left(\Omega_{+}\right) & =\left\{\varphi \in H^{1}\left(\Omega_{+}\right) \mid \varphi=0 \text { on } \Gamma_{+},\left.\varphi\right|_{\Sigma} \in H_{0}^{1}(\Sigma)\right\} \\
H^{1}\left(\Omega_{+}\right) & =\left\{\varphi \in L^{2}\left(\Omega_{+}\right)|\varphi|_{\Sigma} \in L^{2}(\Sigma)\right\} .
\end{aligned}
$$

We will need the following lemma, see [9].

Lemma 2. Let I be a bounded interval of $\mathbb{R}, p \in[1,+\infty]$ and $u \in W^{1, p}(I)$. Then, there exists $\widetilde{u} \in C^{0}(I)$ such that

$$
u=\left.\widetilde{u}\right|_{I} \quad \text { and } \widetilde{u}(b)-\widetilde{u}(a)=\int_{a}^{b} u^{\prime}(t) d t
$$

for all $a, b \in I$.

Proposition 4. If $(u, \theta)$ is the weak limit of $\left(u^{\varepsilon}, \theta^{\varepsilon}\right)$, then

(i) $u_{+} \in L^{\infty}\left(0, T ; \mathcal{V}\left(\Omega_{+}\right)\right), \partial_{t} u_{+} \in L^{\infty}\left(0, T ; \mathcal{H}^{1}\left(\Omega_{+}\right)\right)$and

$$
\begin{cases}u_{1-}=\left.u_{1+}\right|_{\Sigma} & \text { on } \Omega_{-} ; \\ u_{2-}=\left.u_{2+}\right|_{\Sigma} & \text { on } \Omega_{-} ; \\ u_{3-}=0 & \text { on } \Omega_{-} .\end{cases}
$$

(ii) $\theta_{+} \in L^{\infty}\left(0, T ; H^{1}\left(\Omega_{+}\right)\right) \cap L^{2}\left(0, T ; V\left(\Omega_{+}\right)\right)$and $\theta_{-}=\left.\theta_{+}\right|_{\Sigma}$ on $\Omega_{-}$.

\section{Proof.}

(i) Thanks to (4.1), (4.2) and (4.3), we deduce by passing to the limit that $u_{1-}$ and $u_{2-}$ depend only of $x$ and $y$ on $\Omega_{-}$and $u_{3-}$ is independent of $x, y$ and $z$ on $\Omega_{-}$. 
Since $u(., ., z) \in W^{1,2}(]-1,0[)$ and thanks to Lemma 2, there exists a continuous extension $\tilde{u}$ of $u$ to $[-1,0]$. In addition,

$$
\forall Z \in[-1,0]: \quad \widetilde{u}(., ., 0)-\widetilde{u}(., ., Z)=\int_{Z}^{0} \partial_{z} u(., ., t) d t .
$$

As $\int_{Z}^{0} \partial_{z} u(., ., t) d t$ vanishes, it follows that

$$
\widetilde{u}(., ., Z)=\widetilde{u}(., ., 0)
$$

which means that $\widetilde{u}$ on $[-1,0]$ is determined by its value $\widetilde{u}(., ., 0)$ and then $u$ is also determined by its value at the boundary.

(ii) Similarly, we also establish the results on $\theta_{+}$and $\theta_{-}$.

\section{PASSAGe TO THE Limit}

In this section we will show that the asymptotic action (when $\varepsilon \rightarrow 0$ ) of the thin shell $\Omega_{-}^{\varepsilon}$ on the solid $\Omega_{+}$is modelled by evolutionary boundary conditions on the face $\Sigma$ of the boundary of $\Omega_{+}$. Theses boundary conditions are of Ventcel type.

\subsection{Weak formulation of the Ventcel's problem}

Under some conditions, the solution $\left(u^{\varepsilon}, \theta^{\varepsilon}\right)$ of the problem $\left(P^{\varepsilon}\right)$ converges in the weak sense to a limit $(u, \theta)$, itself solution of a limit problem $(P)$.

Proposition 5. Suppose that

(i) $\left(f_{+}^{\varepsilon}, \int_{-1}^{0} f_{1}^{\varepsilon}(., ., z) d z, \int_{-1}^{0} f_{2}^{\varepsilon}(., ., z) d z\right)$ converges weakly to $\left(f_{+}, f^{*}, g^{*}\right)$ in $\mathcal{L}^{2}\left(0, T ; \mathcal{L}^{2}\left(\Omega_{+}\right)\right) \times L^{2}(\Sigma) \times L^{2}(\Sigma)$,

(ii) $\left(u_{+}^{\varepsilon 0}, \int_{-1}^{0} u_{1}^{\varepsilon 0}(., ., z) d z, \int_{-1}^{0} u_{2}^{\varepsilon 0}(., ., z) d z\right)$ converges weakly to $\left(u_{+}^{0}, u_{1}^{0}, u_{2}^{0}\right)$ in $\mathcal{H}^{1}\left(\Omega_{+}\right) \times H^{1}(\Sigma) \times H^{1}(\Sigma)$,

(iii) $\left(u_{+}^{\varepsilon 1}, \int_{-1}^{0} u_{1}^{\varepsilon 1}(., ., z) d z, \int_{-1}^{0} u_{2}^{\varepsilon 1}(., ., z) d z\right)$ converges weakly to $\left(u_{+}^{1}, u_{1}^{1}, u_{2}^{1}\right)$ in $\mathcal{L}^{2}\left(\Omega_{+}\right) \times L^{2}(\Sigma) \times L^{2}(\Sigma)$,

(iv) $\left(g_{+}^{\varepsilon}, \int_{-1}^{0} g^{\varepsilon}(., ., z) d z\right)$ converges weakly to $\left(g_{+}, g\right)$ in $L^{2}\left(0, T ; L^{2}\left(\Omega_{+}\right)\right) \times L^{2}(\Sigma)$,

(v) $\left(\theta_{+}^{\varepsilon 0}, \int_{-1}^{0} \theta^{\varepsilon 0}(., ., z) d z\right)$ converges weakly to $\left(\theta_{+}^{0}, \theta^{1}\right)$ in $L^{2}\left(\Omega_{+}\right) \times L^{2}(\Sigma)$,

then,

(a) $\left(u_{+}^{\varepsilon}, \int_{-1}^{0} u_{1}^{\varepsilon}(., ., z) d z, \int_{-1}^{0} u_{2}^{\varepsilon}(., ., z) d z\right)$ converges weakly-* to $\left(u_{+}, u_{1}, u_{2}\right)$ in $\mathcal{L}^{\infty}\left(0, T ; \mathcal{H}^{1}\left(\Omega_{+}\right)\right) \times H^{1}(\Sigma) \times H^{1}(\Sigma)$,

(b) $\left(\partial_{t} u_{+}^{\varepsilon}, \int_{-1}^{0} \partial_{t} u_{1}^{\varepsilon}(., ., z) d z, \int_{-1}^{0} \partial_{t} u_{2}^{\varepsilon}(., ., z) d z\right)$ converges weakly-* to $\left(\partial_{t} u_{+}, \partial_{t} u_{1}, \partial_{t} u_{2}\right)$ in $\mathcal{L}^{2}\left(0, T ; \mathcal{L}^{2}\left(\Omega_{+}\right)\right) \times L^{2}(\Sigma) \times L^{2}(\Sigma)$

(c) $\left(\theta_{+}^{\varepsilon}, \int_{-1}^{0} \theta^{\varepsilon}(., ., z) d z\right)$ converges weakly-* to $\left(\theta_{+}, \theta\right)$ in $L^{\infty}\left(0, T ; H^{1}\left(\Omega_{+}\right)\right) \times H^{1}(\Sigma)$. 
In addition, $(u, \theta)$ is a weak solution of the following problem $(P)$ : to find

$$
\begin{gathered}
u \in L^{\infty}\left(0, T ; \mathcal{V}\left(\Omega_{+}\right)\right): \partial_{t} u \in L^{\infty}\left(0, T ; \mathcal{H}^{1}\left(\Omega_{+}\right)\right) \\
\text {and } \theta \in L^{\infty}\left(0, T ; L^{2}\left(\Omega_{+}\right)\right) \cap L^{2}\left(0, T ; V\left(\Omega_{+}\right)\right)
\end{gathered}
$$

such that

$$
\begin{aligned}
& \frac{d}{d t}\left(\left\langle\partial_{t} u, v\right\rangle_{+}+\left\langle\partial_{t} u_{1}, v_{1}\right\rangle_{\Sigma}+\left\langle\partial_{t} u_{2}, v_{2}\right\rangle_{\Sigma}\right)+\langle\nabla u, \nabla v\rangle_{+}+\left\langle\partial_{x} u_{1}, \partial_{x} v_{1}\right\rangle_{\Sigma} \\
& +\left\langle\partial_{y} u_{1}, \partial_{y} v_{1}\right\rangle_{\Sigma}+\left\langle\partial_{x} u_{2}, \partial_{x} v_{2}\right\rangle_{\Sigma}+\left\langle\partial_{y} u_{2}, \partial_{y} v_{2}\right\rangle_{\Sigma}+\frac{d}{d t}\left(\langle\theta, \varphi\rangle_{+}+\langle\theta, \varphi\rangle_{\Sigma}\right) \\
& +\langle\nabla \theta, \nabla \varphi\rangle_{+}+\left\langle\partial_{x} \theta, \partial_{x} \varphi\right\rangle_{\Sigma}+\left\langle\partial_{y} \theta, \partial_{y} \varphi\right\rangle_{\Sigma}+\alpha\langle\nabla \theta, v\rangle_{+}+\alpha\left\langle\partial_{x} \theta, v_{1}\right\rangle_{\Sigma} \\
& +\alpha\left\langle\partial_{y} \theta, v_{2}\right\rangle_{\Sigma}-\alpha\left\langle\partial_{t} u, \nabla \varphi\right\rangle_{+}-\alpha\left\langle\partial_{t} u_{1}, \partial_{x} \varphi\right\rangle_{\Sigma}-\alpha\left\langle\partial_{t} u_{2}, \partial_{y} \varphi\right\rangle_{\Sigma} \\
& =\langle f, v\rangle_{+}+\left\langle f_{1}, v_{1}\right\rangle_{\Sigma}+\left\langle f_{2}, v_{2}\right\rangle_{\Sigma}+\langle g, \varphi\rangle_{+}+\langle g, \varphi\rangle_{\Sigma}
\end{aligned}
$$

with

$$
\begin{aligned}
\langle u(0), v\rangle_{+}+\left\langle u_{1}(0), v_{1}\right\rangle_{\Sigma}+\left\langle u_{2}(0), v_{2}\right\rangle_{\Sigma} & =\left\langle u^{0}, v\right\rangle_{+}+\left\langle u_{1}^{0}, v_{1}\right\rangle_{\Sigma}+\left\langle u_{2}^{0}, v_{2}\right\rangle_{\Sigma} \\
\left\langle\partial_{t} u(0), v\right\rangle_{+}+\left\langle\partial_{t} u_{1}(0), v_{1}\right\rangle_{\Sigma}+\left\langle\partial_{t} u_{2}(0), v_{2}\right\rangle_{\Sigma} & =\left\langle u^{1}, \vec{v}\right\rangle_{+}+\left\langle u_{1}^{1}, v_{1}\right\rangle_{\Sigma}+\left\langle u_{2}^{1}, v_{2}\right\rangle_{\Sigma} \\
\langle\theta(0), \varphi\rangle_{+}+\langle\theta(0), \varphi\rangle_{\Sigma} & =\left\langle\theta^{0}, \varphi\right\rangle_{+}+\left\langle\theta^{0}, \varphi\right\rangle_{\Sigma}
\end{aligned}
$$

for all $(v, \varphi) \in \mathcal{V}(\Omega) \times V(\Omega)$.

Proof. It suffices to note that the weak formulation is as follows:

$$
\begin{aligned}
& \frac{d}{d t}\left(\left\langle\partial_{t} u^{\varepsilon}, \vec{v}^{\varepsilon}\right\rangle_{+}+\left\langle\partial_{t} u_{1}^{\varepsilon}, v_{1}^{\varepsilon}\right\rangle_{-}+\left\langle\partial_{t} u_{2}^{\varepsilon}, v_{2}^{\varepsilon}\right\rangle_{-}+\frac{1}{\varepsilon^{2}}\left\langle\partial_{t} u_{3}^{\varepsilon}, v_{3}^{\varepsilon}\right\rangle_{-}\right) \\
& +\left\langle\nabla u^{\varepsilon}, \nabla v^{\varepsilon}\right\rangle_{+}+\left\langle\partial_{x} u_{1}^{\varepsilon}, \partial_{x} v_{1}^{\varepsilon}\right\rangle_{-}+\left\langle\partial_{y} u_{1}^{\varepsilon}, \partial_{y} v_{1}^{\varepsilon}\right\rangle_{-}+\frac{1}{\varepsilon^{2}}\left\langle\partial_{z} u_{1}^{\varepsilon}, \partial_{z} v_{1}^{\varepsilon}\right\rangle_{-} \\
& +\left\langle\partial_{x} u_{2}^{\varepsilon}, \partial_{x} v_{2}^{\varepsilon}\right\rangle_{-}+\left\langle\partial_{y} u_{2}^{\varepsilon}, \partial_{y} v_{2}^{\varepsilon}\right\rangle_{-}+\frac{1}{\varepsilon^{2}}\left\langle\partial_{z} u_{2}^{\varepsilon}, \partial_{z} v_{2}^{\varepsilon}\right\rangle_{-}+\frac{1}{\varepsilon^{2}}\left\langle\partial_{x} u_{3}^{\varepsilon}, \partial_{x} v_{3}^{\varepsilon}\right\rangle_{-} \\
& +\frac{1}{\varepsilon^{2}}\left\langle\partial_{y} u_{3}^{\varepsilon}, \partial_{y} v_{3}^{\varepsilon}\right\rangle_{-}+\frac{1}{\varepsilon^{4}}\left\langle\partial_{z} u_{3}^{\varepsilon}, \partial_{z} v_{3}^{\varepsilon}\right\rangle_{-}+\frac{d}{d t}\left(\left\langle\theta^{\varepsilon}, \varphi^{\varepsilon}\right\rangle_{+}+\left\langle\theta^{\varepsilon}, \varphi^{\varepsilon}\right\rangle_{-}\right) \\
& +\left\langle\nabla \theta^{\varepsilon}, \nabla \varphi^{\varepsilon}\right\rangle_{+}+\left\langle\partial_{x} \theta^{\varepsilon}, \partial_{x} \varphi^{\varepsilon}\right\rangle_{-}+\left\langle\partial_{y} \theta^{\varepsilon}, \partial_{y} \varphi^{\varepsilon}\right\rangle_{-}+\frac{1}{\varepsilon^{2}}\left\langle\partial_{z} \theta^{\varepsilon}, \partial_{z} \varphi^{\varepsilon}\right\rangle_{-} \\
& +\alpha\left\langle\nabla \theta^{\varepsilon}, v^{\varepsilon}\right\rangle_{+}+\alpha\left\langle\partial_{x} \theta^{\varepsilon}, v_{1}^{\varepsilon}\right\rangle_{-}+\alpha\left\langle\partial_{y} \theta^{\varepsilon}, v_{2}^{\varepsilon}\right\rangle_{-}+\frac{1}{\varepsilon^{2}} \alpha\left\langle\partial_{z} \theta^{\varepsilon}, v_{3}^{\varepsilon}\right\rangle_{-} \\
& -\alpha\left\langle\partial_{t} u^{\varepsilon}, \nabla \varphi^{\varepsilon}\right\rangle_{+}-\alpha\left\langle\partial_{t} u_{1}^{\varepsilon}, \partial_{x} \varphi^{\varepsilon}\right\rangle_{-}-\alpha\left\langle\partial_{t} u_{2}^{\varepsilon}, \partial_{y} \varphi^{\varepsilon}\right\rangle_{-}-\frac{1}{\varepsilon^{2}} \alpha\left\langle\partial_{t} u_{3}^{\varepsilon}, \partial_{z} \varphi^{\varepsilon}\right\rangle_{-} \\
& =\left\langle f^{\varepsilon}, v^{\varepsilon}\right\rangle_{+}+\left\langle f_{1}^{\varepsilon}, v_{1}^{\varepsilon}\right\rangle_{-}+\left\langle f_{2}^{\varepsilon}, v_{2}^{\varepsilon}\right\rangle_{-}+\frac{1}{\varepsilon^{2}}\left\langle f_{3}^{\varepsilon}, v_{3}^{\varepsilon}\right\rangle_{-}+\left\langle g^{\varepsilon}, \varphi^{\varepsilon}\right\rangle_{+}+\left\langle g^{\varepsilon}, \varphi^{\varepsilon}\right\rangle_{-} .
\end{aligned}
$$


As we have (see, [9])

$$
\iiint_{\Omega_{-}} \ldots d x d y d z=\int_{-1}^{0}\left(\iint_{\Sigma} \ldots d x d y\right) d z=\iint_{\Sigma}\left(\int_{-1}^{0} \ldots d z\right) d x d y
$$

then the weak formulation becomes:

$$
\begin{aligned}
& \frac{d}{d t}\left(\left\langle\partial_{t} u^{\varepsilon}, v^{\varepsilon}\right\rangle_{+}+\int_{-1}^{0}\left(\iint_{\Sigma} \partial_{t} u_{1}^{\varepsilon} v_{1-}^{\varepsilon} d x d y\right) d z+\int_{-1}^{0}\left(\iint_{\Sigma} \partial_{t} u_{2}^{\varepsilon} v_{2-}^{\varepsilon} d x d y\right) d z\right. \\
& \left.+\frac{1}{\varepsilon^{2}} \int_{-1}^{0}\left(\iint_{\Sigma} \partial_{t} u_{3}^{\varepsilon} v_{3}^{\varepsilon} d x d y\right) d z\right)+\left\langle\nabla u^{\varepsilon}, \nabla v^{\varepsilon}\right\rangle_{+}+\int_{-1}^{0}\left(\iint_{\Sigma} \partial_{x} u_{1}^{\varepsilon} \partial_{x} v_{1}^{\varepsilon} d x d y\right) d z \\
& +\int_{-1}^{0}\left(\iint_{\Sigma} \partial_{y} u_{1}^{\varepsilon} \partial_{y} v_{1}^{\varepsilon} d x d y\right) d z+\frac{1}{\varepsilon^{2}} \int_{-1}^{0}\left(\iint_{\Sigma} \partial_{z} u_{1}^{\varepsilon} \partial_{z} v_{1}^{\varepsilon} d x d y\right) d z \\
& +\int_{-1}^{0}\left(\iint_{\Sigma} \partial_{x} u_{2}^{\varepsilon} \partial_{x} v_{2}^{\varepsilon} d x d y\right) d z+\int_{-1}^{0}\left(\iint_{\Sigma} \partial_{y} u_{2}^{\varepsilon} \partial_{y} v_{2}^{\varepsilon} d x d y\right) d z \\
& +\frac{1}{\varepsilon^{2}} \int_{-1}^{0}\left(\iint_{\Sigma} \partial_{z} u_{2}^{\varepsilon} \partial_{z} v_{2}^{\varepsilon} d x d y\right) d z+\frac{1}{\varepsilon^{2}} \int_{-1}^{0}\left(\iint_{\Sigma} \partial_{x} u_{3}^{\varepsilon} \partial_{x} v_{3}^{\varepsilon} d x d y\right) d z \\
& +\frac{1}{\varepsilon^{2}} \int_{-1}^{0}\left(\iint_{\Sigma} \partial_{y} u_{3}^{\varepsilon} \partial_{y} v_{3}^{\varepsilon} d x d y\right) d z+\frac{1}{\varepsilon^{4}} \int_{-1}^{0}\left(\iint_{\Sigma} \partial_{z} u_{3}^{\varepsilon} \partial_{z} v_{3}^{\varepsilon} d x d y\right) d z \\
& +\frac{d}{d t}\left(\left\langle\theta^{\varepsilon}, \varphi^{\varepsilon}\right\rangle_{+}+\int_{-1}^{0}\left(\iint_{\Sigma} \theta^{\varepsilon} \varphi^{\varepsilon} d x d y\right) d z\right)+\left\langle\nabla \theta^{\varepsilon}, \nabla \varphi^{\varepsilon}\right\rangle_{+} \\
& +\int_{-1}^{0}\left(\iint_{\Sigma} \partial_{x} \theta^{\varepsilon} \partial_{x} \varphi^{\varepsilon} d x d y\right) d z+\int_{-1}^{0}\left(\iint_{\Sigma} \partial_{y} \theta^{\varepsilon} \partial_{y} \varphi^{\varepsilon} d x d y\right) d z \\
& +\frac{1}{\varepsilon^{2}} \int_{-1}^{0}\left(\iint_{\Sigma} \partial_{z} \theta^{\varepsilon} \partial_{z} \varphi^{\varepsilon} d x d y\right) d z+\alpha\left\langle\nabla \theta^{\varepsilon}, v^{\varepsilon}\right\rangle_{+}+\alpha \int_{-1}^{0}\left(\iint_{\Sigma} \partial_{x} \theta^{\varepsilon} v_{1}^{\varepsilon} d x d y\right) d z \\
& +\alpha \int_{-1}^{0}\left(\iint_{\Sigma} \partial_{y} \theta^{\varepsilon} v_{2}^{\varepsilon} d x d y\right) d z+\frac{\alpha}{\varepsilon^{2}} \int_{-1}^{0}\left(\iint_{\Sigma} \partial_{z} \theta^{\varepsilon} v_{3}^{\varepsilon} d x d y\right) d z-\alpha\left\langle\partial_{t} u^{\varepsilon}, \nabla \varphi^{\varepsilon}\right\rangle_{+}
\end{aligned}
$$


594

NASSIM BOUDRAHEM AND AHMED BERBOUCHA

$$
\begin{aligned}
& -\alpha \int_{-1}^{0}\left(\iint_{\Sigma} \partial_{t} u_{1}^{\varepsilon} \partial_{x} \varphi^{\varepsilon} d x d y\right) d z-\alpha \int_{-1}^{0}\left(\iint_{\Sigma} \partial_{t} u_{2}^{\varepsilon} \partial_{y} \varphi^{\varepsilon} d x d y\right) d z \\
& -\frac{\alpha}{\varepsilon^{2}} \int_{-1}^{0}\left(\iint_{\Sigma} \partial_{t} u_{3}^{\varepsilon} \partial_{z} \varphi^{\varepsilon} d x d y\right) d z \\
& =\left\langle f^{\varepsilon}, v^{\varepsilon}\right\rangle_{+}+\int_{-1}^{0}\left(\iint_{\Sigma} f_{1}^{\varepsilon} v_{1}^{\varepsilon} d x d y\right) d z+\int_{-1}^{0}\left(\iint_{\Sigma} f_{2}^{\varepsilon} v_{2}^{\varepsilon} d x d y\right) d z \\
& +\frac{1}{\varepsilon^{2}} \int_{-1}^{0}\left(\iint_{\Sigma} f_{3}^{\varepsilon} v_{3}^{\varepsilon} d x d y\right) d z+\left\langle g^{\varepsilon}, \varphi^{\varepsilon}\right\rangle_{+}+\int_{-1}^{0}\left(\iint_{\Sigma} g^{\varepsilon} \varphi^{\varepsilon} d x d y\right) d z .
\end{aligned}
$$

We take then $v^{\varepsilon}$ and $\varphi^{\varepsilon}$ such that

$v_{+}^{\varepsilon} \in \mathcal{V}\left(\Omega_{+}\right)=\left\{v \in \mathcal{H}^{1}\left(\Omega_{+}\right) \mid v=0\right.$ on $\Gamma_{+}, v_{1} \in H_{0}^{1}(\Sigma), v_{2} \in H_{0}^{1}(\Sigma), v_{3}=0$ on $\left.\Sigma\right\}$

$v_{-}^{\varepsilon}$ as follows:

$$
\begin{gathered}
\begin{cases}v_{1-}^{\varepsilon}=\left.v_{1+}\right|_{\Sigma} & \text { on } \Omega_{-} ; \\
v_{2-}^{\varepsilon}=\left.v_{2+}\right|_{\Sigma} & \text { on } \Omega_{-} ; \\
v_{3-}^{\varepsilon}=0 & \text { on } \Omega_{-} ;\end{cases} \\
\varphi_{+}^{\varepsilon} \in V\left(\Omega_{+}\right)=\left\{\varphi \in H^{1}\left(\Omega_{+}\right) \mid \varphi=0 \text { on } \Gamma_{+},\left.\varphi\right|_{\Sigma} \in H_{0}^{1}(\Sigma)\right\}
\end{gathered}
$$

and

$$
\varphi_{-}^{\varepsilon}=\left.\varphi_{+}^{\varepsilon}\right|_{\Sigma} \text { on } \Omega_{-} .
$$

Then, we obtain

$$
\begin{aligned}
& \frac{d}{d t}\left(\left\langle\partial_{t} u^{\varepsilon}, v^{\varepsilon}\right\rangle_{+}+\iint_{\Sigma}\left(\int_{-1}^{0} \partial_{t} u_{1}^{\varepsilon} d z\right) v_{1-}^{\varepsilon} d x d y+\iint_{\Sigma}\left(\int_{-1}^{0} \partial_{t} u_{2}^{\varepsilon} d z\right) v_{2-}^{\varepsilon} d x d y\right) \\
& +\left\langle\nabla u^{\varepsilon}, \nabla v^{\varepsilon}\right\rangle_{+}+\iint_{\Sigma}\left(\int_{-1}^{0} \partial_{x} u_{1}^{\varepsilon} d z\right) \partial_{x} v_{1}^{\varepsilon} d x d y+\iint_{\Sigma}\left(\int_{-1}^{0} \partial_{y} u_{1}^{\varepsilon} d z\right) \partial_{y} v_{1}^{\varepsilon} d x d y \\
& +\iint_{\Sigma}\left(\int_{-1}^{0} \partial_{x} u_{2}^{\varepsilon} d z\right) \partial_{x} v_{2}^{\varepsilon} d x+\iint_{\Sigma}\left(\int_{-1}^{0} \partial_{x} u_{1}^{\varepsilon} d z\right) \partial_{x} v_{1}^{\varepsilon} d x d y \\
& +\iint_{\Sigma}\left(\int_{-1}^{0} \partial_{y} u_{1}^{\varepsilon} d z\right) \partial_{y} v_{1}^{\varepsilon} d x d y+\iint_{\Sigma}\left(\int_{-1}^{0} \partial_{x} u_{2}^{\varepsilon} d z\right) \partial_{x} v_{2}^{\varepsilon} d x d y \\
& +\iint_{\Sigma}\left(\int_{-1}^{0} \partial_{y} u_{2}^{\varepsilon} d z\right) \partial_{y} v_{2}^{\varepsilon} d x d y+\frac{d}{d t}\left(\left\langle\theta^{\varepsilon}, \varphi^{\varepsilon}\right\rangle_{+}+\iint_{\Sigma}\left(\int_{-1}^{0} \theta^{\varepsilon} d z\right) \varphi^{\varepsilon} d x d y\right)
\end{aligned}
$$




$$
\begin{aligned}
& +\left\langle\nabla \theta^{\varepsilon}, \nabla \varphi^{\varepsilon}\right\rangle_{+}+\iint_{\Sigma}\left(\int_{-1}^{0} \partial_{x} \theta^{\varepsilon} d z\right) \partial_{x} \varphi^{\varepsilon} d x d y+\iint_{\Sigma}\left(\int_{-1}^{0} \partial_{y} \theta^{\varepsilon} d z\right) \partial_{y} \varphi^{\varepsilon} d x d y \\
& +\alpha\left\langle\nabla \theta^{\varepsilon}, v^{\varepsilon}\right\rangle_{+}+\alpha \iint_{\Sigma}\left(\int_{-1}^{0} \partial_{x} \theta^{\varepsilon} d z\right) v_{1}^{\varepsilon} d x d y+\alpha \iint_{\Sigma}\left(\int_{-1}^{0} \partial_{y} \theta^{\varepsilon} d z\right) v_{2}^{\varepsilon} d x d y \\
& -\alpha\left\langle\partial_{t} u^{\varepsilon}, \nabla \varphi^{\varepsilon}\right\rangle_{+}-\alpha \iint_{\Sigma}\left(\int_{-1}^{0} \partial_{t} u_{1}^{\varepsilon} d z\right) \partial_{x} \varphi^{\varepsilon} d x d y \\
& -\alpha \iint_{\Sigma}\left(\int_{-1}^{0} \partial_{t} u_{2}^{\varepsilon} d z\right) \partial_{y} \varphi^{\varepsilon} d x d y \\
& =\left\langle f^{\varepsilon}, v^{\varepsilon}\right\rangle_{+}+\iint_{\Sigma}\left(\int_{-1}^{0} f_{1}^{\varepsilon} d z\right) v_{1}^{\varepsilon} d x d y+\iint_{\Sigma}\left(\int_{-1}^{0} f_{2}^{\varepsilon} d z\right) v_{2}^{\varepsilon} d x d y+ \\
& \left\langle g^{\varepsilon}, \varphi^{\varepsilon}\right\rangle_{+}+\iint_{\Sigma}\left(\int_{-1}^{0} g^{\varepsilon} d z\right) \varphi^{\varepsilon} d x d y
\end{aligned}
$$

and we deduce the result by passing to the limit.

\subsection{Strong formulation of the Ventcel's problem}

Under the conditions of Proposition 5 and by using an integration by parts on the boundary, the obtained weak problem can be expressed as follows:

$$
\begin{cases}u_{+}^{\prime \prime}-\triangle u_{+}+\alpha \nabla \theta_{+}=f_{+} & \text {on } \Omega_{+} \times(0, T) ; \\ \theta_{+}^{\prime}-\triangle \theta_{+}+\alpha \operatorname{div}\left(u_{+}^{\prime}\right)=g_{+} & \text {on } \Omega_{+} \times(0, T) ;\end{cases}
$$

with Dirichlet conditions on the fixed portion of the boundary $\Gamma_{+}$

$$
u_{+}=0, \quad \theta_{+}=0 \quad \text { on } \Gamma_{+} \times(0, T),
$$

Ventcel type conditions on the side of junction $\Sigma$

$$
\begin{cases}u_{1}^{\prime \prime}-\partial_{x^{2}}^{2} u_{1}-\partial_{y^{2}}^{2} u_{1}-\partial_{z} u_{1+}+\alpha \partial_{x} \theta=f_{1} & \text { on } \Sigma \times(0, T) ; \\ u_{2}^{\prime \prime}-\partial_{x^{2}}^{2} u_{2}-\partial_{y^{2}}^{2} u_{2}-\partial_{z} u_{2+}+\alpha \partial_{y} \theta=f_{2} & \text { on } \Sigma \times(0, T) ; \\ u_{3}=0 & \text { on } \Sigma \times(0, T) ; \\ \theta^{\prime}-\partial_{x^{2}}^{2} \theta-\partial_{y^{2}}^{2} \theta-\partial_{z} \theta_{+}+\alpha \partial_{x} u_{1}^{\prime}+\alpha \partial_{y} u_{2}^{\prime}=g & \text { on } \Sigma \times(0, T) ;\end{cases}
$$

and Cauchy initial conditions

$$
u_{+}(0)=u_{+}^{0}, \quad u_{+}^{\prime}(0)=u_{+}^{1}, \quad \theta_{+}(0)=\theta_{+}^{0} \text { on } \Omega_{+}
$$

and

$$
u_{1}(0)=u_{1}^{0}, \quad u_{2}(0)=u_{2}^{0}, \quad u_{1}^{\prime}(0)=u_{1}^{1}, \quad u_{2}^{\prime}(0)=u_{2}^{1}, \quad \theta(0)=\theta_{1} \text { on } \Sigma .
$$




\section{CONCLUSION}

The model thus constructed has, on the face covered with a thin shell, boundary conditions involving tangential and time derivatives of the same order as that of the interior differential operator. These conditions are called of Ventcel type.

This work allows also to have a reference in three-dimensional case for approximate boundary conditions as effect of a thin layer.

\section{ACKNOWLEDGEMENT}

We acknowledge support of "Direction Générale de la Recherche Scientifique et du Développement Technologique (DGRSDT)". MESRS, Algeria.

We are grateful to anonymous reviewers for carefully reading this paper and for their valuable comments and suggestions, which have improved the paper.

\section{REFERENCES}

[1] M. V. Abdelkader and A. A. Moussa, "Asymptotic study of thin elastic layer." Applied Mathematical Sciences, vol. 7, no. 108, pp. 5385-5396, 2013, doi: 10.12988/ams.2013.36331.

[2] E. Acerbi and G. Buttazzo, "Reinforcement problems in the calculus of variations." Ann. Inst. H. Poincaré(C) Non linear Analysis, vol. 3, no. 4, pp. 273-284, 1986, doi: 10.1016/S02941449(16)30380-8.

[3] R. P. Agarwal, S. Gala, and M. A. Ragusa, "A regularity criterion of the 3D MHD equations involving one velocity and one current density component in Lorentz space." Zeitschrift fur angewandte mathematik und physik, vol. 71, no. 95, 2020, doi: 10.1007/s00033-020-01318-4.

[4] H. Ammari, E. Beretta, and F. Francini, "Reconstruction of thin conductivity imperfections." Applicable Analysis, vol. 83, no. 1, pp. 63-76, 2004, doi: 10.1080/00036810310001620090.

[5] A. Auvray and G. Vial, "Asymptotic expansions and effective boundary conditions: A short review for smooth and nonsmooth geometries with thin layers." Essaim: Proceeding and surveys, vol. 61, pp. 38-54, 2018, doi: 10.1051/proc/201861038.

[6] A. Auvray and G. Vial, "Improved impedance conditions for a thin layer problem in a non smooth domain." Math. Meth. Appl. Sci., vol. 42, no. 5, pp. 1432-1448, 2019, doi: 10.1002/mma.5434.

[7] S. Benbernou, S. Gala, and M. A. Ragusa, "On the regularity criteria for the 3D magnetohydrodynamic equations via two components in terms of BMO space." Mathematical Methods in the Applied Sciences, vol. 37, no. 15, pp. 2320-2325, 2014, doi: 10.1002/mma.2981.

[8] A. Bendali and K. Lemrabet, "The effect of a thin coating on the scattering of a time-harmonic wave for the Helmholtz equation." SIAM J. Appl. Math., vol. 56, no. 6, pp. 1664-1693, 1996, doi: 10.1137/S0036139995281822.

[9] H. Brezis, Opérateurs Maximaux Monotones et Semi-Groupes de Contractions dans les Espaces de Hilbert. North-Holland Mathematics Studies, 1973.

[10] Y. Capdeville and J. J. Marigo, "Shallow layer correction for spectral element like methods." Geophys. J. Int., vol. 172, pp. 1135-1150, 2008, doi: 10.1111/j.1365-246X.2007.03703.x.

[11] B. Delourme, H. Haddar, and P. Joly, "Approximate models for wave propagation across thin periodic interfaces." J. Math. Pures Appl., vol. 98, no. 1, pp. 28-71, 2012, doi: 10.1016/j.matpur.2012.01.003.

[12] B. Engquist and A. Majda, "Absorbing boundary conditions for the numerical simulation of waves.” Math. Comp., vol. 31, pp. 629-651, 1977, doi: 10.1090/S0025-5718-1977-0436612-4. 
[13] B. Engquist and J. C. Nedelec, "Effective boundary conditions for electromagnetic scattering in thin layers." Rapport interne CMAP, vol. 278, 1993.

[14] G. Geymonat, F. Krasucki, and S. Lenci, "Mathematical analysis of a bonded joint with a soft thin adhesive." Math. Mech. Solids, vol. 4, no. 2, pp. 201-225, 1999, doi: 10.1177/108128659900400204.

[15] D. Givoli, "Finite element modeling of thin layers." Comput. Model. Eng. Sci., vol. 5, no. 6, pp. 497-514, 2004, doi: 10.3970/cmes.2004.005.497.

[16] H. Haddar and P. Joly, "Stability of thin layer approximation of electromagnetic waves scattering by linear and nonlinear coatings." Journal of Computational and Applied Mathematics, vol. 143, no. 2, pp. 201-236, 2002, doi: 10.1016/S0377-0427(01)00508-8.

[17] V. V. Hung and H. N. Quy, "The m-Hessian operator on some weighted energy classes of delta m-subharmonic functions." Results in Mathematics, vol. 75, no. 112, 2020, doi: 10.1007/s00025020-01242-z.

[18] J. L. Lions and E. Magenes, Problèmes aux Limites Non Homogènes et Applications. Paris: Dunod, 1970.

[19] P. Milosevic, E. Milovanovic, M. Matejic, and I. Milovanovic, "On relations between Kirchhoff index, Laplacian energy, Laplacian-energy-like invariant and degree deviation of graphs." Filomat, vol. 34, no. 3, pp. 1025-1033, 2020, doi: 10.2298/FIL2003025M.

[20] J. Necas, Les Méthodes Directes en Théorie des Équations Elliptiques. Paris: Masson, 1967.

[21] M. A. Ragusa and A. Tachikawa, "Partial regularity of the minimizers of quadratic functionals with VMO coefficients." Journal of the London Mathematical Society, vol. 72, no. 2, pp. 609620, 2005, doi: 10.1112/S002461070500699X.

[22] L. Rahmani, "Ventcel's boundary conditions for a dynamic nonlinear plate." Asymptotic Analysis, vol. 38, no. 3-4, pp. 319-337, 2004.

[23] L. Rahmani, "The effect of thin layer on a nonlinear thermoelastistic plate." Applied Mathematical Sciences, vol. 2, no. 50, pp. 2489-2499, 2008.

[24] L. Rahmani, "Modelling of the effect of a thin stiffener on the boundary of a nonlinear thermoelastic plate." Mathematical Modelling and Analysis, vol. 14, no. 3, pp. 353-369, 2009, doi: 10.3846/1392-6292.2009.14.353-368.

[25] L. Rahmani, "Modeling of the nonlinear vibrations of a stiffened moderately thick plate." $C . R$. Acad. Sci., vol. Ser. I, no. 352, pp. 223-227, 2014, doi: 10.1016/j.crma.2014.01.002.

[26] H. Reinhard, Equations Différentielles: Fondements et Applications. Gauthier-Villars, 1982.

[27] E. Wȩgrzyn-Skrzypczak, "Discontinuous Galerkin method for the three-dimensional problem of thermoelasticity." Journal of Applied Mathematics and Computational Mechanics, vol. 18, no. 4, pp. 115-126, 2019, doi: 10.17512/jamcm.2019.4.11.

[28] E. Wȩgrzyn-Skrzypczak, "Analysis of the three-dimensional thermoelasticity with the use of the continuous Galerkin method." Journal of Applied Mathematics and Computational Mechanics, vol. 19, no. 3, pp. 111-121, 2020, doi: 10.17512/jamcm.2020.3.09.

Authors' addresses

Nassim Boudrahem

Laboratoire de Mathématiques Appliquées, Faculté des Sciences Exactes, Université de Bejaia, 06000, Algérie

E-mail address: nassim.boudrahem@univ-bejaia.dz 
Ahmed Berboucha

(Corresponding author) Laboratoire de Mathématiques Appliquées, Faculté des Sciences Exactes, Université de Bejaia, 06000, Algérie

E-mail address: ahmed.berboucha@univ-bejaia.dz 